$01,10,19$

\title{
Метод определения параметров парного межатомного потенциала
}

\author{
(C) М.Н. Магомедов \\ Институт проблем геотермии и возобновляемой энергетики - \\ филиал Объединенного института высоких температур РАН, \\ Махачкала, Россия \\ E-mail: mahmag4@mail.ru
}

Поступила в Редакцию 11 февраля 2020 г.

В окончательной редакции 11 февраля 2020 г.

Принята к публикации 11 февраля 2020 г.

\begin{abstract}
Указаны недостатки известных из литературы методов определения 4-х параметров парного межатомного потенциала Ми-Леннард-Джонса применительно к кристаллам. Предложен новый метод для определения параметров этого потенциала по термоупругим свойствам кристалла. В данном методе параметры определяются по наилучшему совпадению рассчитанных значений с экспериментальными данными: 1) энергии сублимации кристалла при нулевых значениях температуры $(T=0 \mathrm{~K})$ и давления $(P=0)$; 2$)$ коэффициента теплового расширения и изотермического модуля упругости, измеренными при $P=0$ и $T=300 \mathrm{~K}$; $3)$ зависимостью изотермы $T=300 \mathrm{~K}$ уравнения состояния от объема $P(300 \mathrm{~K}, V)$. Метод был апробирован на железе и золоте и показал хорошие результаты. Данным методом были также определены параметров межатомного потенциала для тугоплавких металлов: $\mathrm{Nb}$, Тa, Мо и $\mathrm{W}$. Полученные результаты позволили также более точнее определить такие свойства данных металлов как энергия сублимации, температура Дебая и поверхностная энергия.
\end{abstract}

Ключевые слова: межатомный потенциал, уравнение состояния, тепловое расширение, поверхностная энергияю

DOI: $10.21883 /$ FTT.2020.07.49462.026

\section{1. Введение}

Для численного или аналитического расчета термодинамических свойств кристалла простого (однокомпонентного) вещества необходимо определить потенциал взаимодействия пары его атомов. Для аналитического расчета часто парное межатомное взаимодействие в кристалле простого вещества представляется в виде 4-х параметрического потенциала Ми-Леннард-Джонса, который имеет следующий вид [1-7]:

$$
\varphi(r)=\frac{D}{(b-a)}\left[a\left(\frac{r_{o}}{r}\right)^{b}-b\left(\frac{r_{o}}{r}\right)^{a}\right],
$$

где $D$ и $r_{o}$ - глубина и координата минимума потенциала, $b>a>1$ - численные параметры.

В литературе встречаются высказывания, что парный 4-х параметрический потенциал Ми-Леннард-Джонca (1) дает при расчетах свойств кристаллов худшие результаты, чем трех параметрический потенциал Морзе для металлов, либо многочастичные потенциалы для $\mathrm{Si}$ или Ge (потенциалы типа Stillinger-Weber или Tersoff, которые включают не менее 10-12 параметров, и которые можно использовать только в численных расчетах). Это мнение возникло из-за трудностей определения самосогласованным образом всех четырех параметров потенциала Ми-Леннард-Джонса, особенно значений степенней $a$ и $b$. Поэтому ранее (лет 50 тому назад) в большинстве расчетов брали ничем не обоснованные значения степенных параметров $a=6$ и $b=12$. Это было обусловлено тем, что с параметрами 6 и 12 получается квадратное уравнение, с которым было легче работать. Потенциал 6-12 использовался Леннард-Джонсом с соавт. в ,докомпьютерную эру“ (т.е. 30-40-х годах $\mathrm{XX}$ века) для описания свойств инертных газов [8]. По этой же причине потенциал 6-12 (названный потенциалом Леннард-Джонса) был применен для расчета свойств твердой фазы металлов и диэлектриков. Это и привело к плохим результатам, на основе которых и сложилось неправильное мнение о неприменимости потенциала Ми-Леннард-Джонса общего вида (1), т. е. со степенями $a-b$, для описания свойств металлов. Отметим, что потенциал Морзе дает для металлов более лучшие результаты чем потенциал Леннард-Джонса 6-12. Но 3-х параметрический потенциал Морзе является частным случаем 4-х параметрического потенциала Ми-Леннард-Джонса (1) при $b=2 a$ [8, Т. 1, стр. 282].

Задача корректного определения параметров потенциала (1) решалась во многих работах (см. обзор в $[1,2])$, но так до сих пор и не получила полного решения. Координата минимума потенциала $r_{o}$ легко вычисляется либо из мольного объема $\left(V_{00}\right)$, либо из параметра решетки $\left(l_{00}\right)$ кристалла при нулевых значениях температуры $T=0 \mathrm{~K}$ и давления $P=0$ по формулам $[1,2]$ :

$r_{o}=\left(q_{v} \frac{V_{00}}{N_{A}}\right)^{1 / 3}, \quad r_{o}=\left(6 \frac{k_{p} V_{00}}{\pi N_{A}}\right)^{1 / 3}, \quad r_{o}=q_{l} l_{00}=c_{00}$.

Здесь $N_{A}-$ число Авогадро, $k_{p}-$ коэффициент упаковки структуры, $c_{00}$ - расстояние между центрами 
ближайших атомов при $T=0 \mathrm{~K}$ и $P=0$, структурные константы $q_{v}$ и $q_{l}$ были определены в $[1,2]$.

Величину $D-$ глубину потенциальной ямы можно определить из молярной энергии сублимации кристалла, экстраполированной к $T=0 \mathrm{~K}$ при $P=0: L_{00}$. В paботе [1] величину $D$ определяли из $L_{00}$ без учета энергии „нулевых колебаний“ решетки кристалла. Но это приближение плохо выполняется даже для металлов. Параметры $b$ и $a$ в [1] определялись из зависимости величины $L_{00} /\left(B_{00} r_{o}^{3}\right)$ от произведения $b a$. Здесь $B=-V(\partial P / \partial V)_{T}-$ изотермический модуль упругости. Такой подход позволил в [1] приближенно рассчитать параметры $r_{o}, D, b$ и $a$ для металлов с различной структурой.

Нами в [2] был предложен метод самосогласованного определения всех 4-х параметров потенциала (1): $r_{o}$, $D, b$ и $a$, который состоит в следующем. Значение $r_{o}$ вычисляется по формулам (2). Величина $D$ в приближении „взаимодействия только ближайших соседей“ вычислялась из уравнения, в котором учитывается энергия „нулевых колебаний“

$$
L_{00} / N_{A}=\left(k_{n} / 2\right) D-(9 / 8) k_{B} \Theta_{00},
$$

где $k_{n}$ - первое координационное число, $k_{B}$ - постоянная Больцмана, $\Theta_{00}-$ температура Дебая при $T=0 \mathrm{~K}$ и $P=0$.

Для определения степеней $b$ и $a$ в [2] используется выражение для $\Theta$ и вытекающая из этого выражения формула для первого параметра Грюнайзена $(\gamma)$, которые были получены в [9] также на основе приближения „взаимодействия только ближайших соседей“

$$
\begin{gathered}
\Theta\left(k_{n}, c\right)=A_{w}\left(k_{n}, c\right) \xi\left[-1+\left(1+\frac{8 D}{k_{B} A_{w}\left(k_{n}, c\right) \xi^{2}}\right)^{1 / 2}\right], \\
\gamma=-\left(\frac{\partial \ln \Theta}{\partial \ln V}\right)_{T}=\frac{b+2}{6\left(1+X_{w}\right)} .
\end{gathered}
$$

Здесь $c=\left[6 k_{p} V /(\pi N)\right]^{1 / 3}-$ расстояние между центрами ближайших атомов, функция $A_{w}$ возникает из-за учета энергии „нулевых колебаний“ атомов в кристалле,

$$
\begin{gathered}
A_{w}\left(k_{n}, c\right)=K_{R} \frac{5 k_{n} a b(b+1)}{144(b-a)}\left(\frac{r_{o}}{c}\right)^{b+2}, \\
K_{R}=\frac{\hbar^{2}}{k_{B} r_{o}^{2} m}, \quad \xi=\frac{9}{k_{n}},
\end{gathered}
$$

где $m-$ масса атома, $\hbar-$ постоянная Планка, функция $X_{w}=A_{w} \xi / \Theta$ определяет роль квантовых эффектов в кристалле.

Таким образом, для одноатомного вещества параметры $r_{o}, D, b$ и $a$ в [2] однозначно определялись из формул (2) и из 3-х уравнений, в которые входят три экспериментально определенные при $T=0 \mathrm{~K}$ и $P=0$ величины: $L_{00}, \Theta_{00}, \gamma_{00}$ :

$$
\begin{gathered}
D / k_{B}=\left(2 / k_{n}\right)\left\{\left[L_{00} /\left(N_{A} k_{B}\right)\right]+\left(9 \Theta_{00} / 8\right)\right\}, \\
b=6 \gamma_{00}\left[1+\left(2 F_{b}\right)^{-1}\right]-2,
\end{gathered}
$$

$$
a=b /\left\{1+\left[5 K_{R} k_{n} b(b+1) /\left(144 A_{w}(1)\right)\right]\right\} .
$$

Здесь, согласно (4) и (6), имеем: $A_{w}(1)=\Theta_{00} /\left(2 \xi F_{b}\right)$, $F_{b}=\left[4 D /\left(k_{B} \xi \Theta_{00}\right)\right]-1$.

Таким методом в [2] были определены параметры потенциала (1) для многих элементарных кристаллов. При этом было учтено, что потенциальные параметры простых веществ ограничены определенными интервалами допустимых значений

$$
0.67 \mathrm{~K} \leq D / k_{B} \leq 97473.6 \mathrm{~K} \text { и } 6 \leq a b \leq 108 .
$$

Но метод из [2] предполагает, что значения $L_{00}$, $\Theta_{00}$ и $\gamma_{00}$ определены в эксперименте при $T=0 \mathrm{~K}$ и $P=0$ с высокой точностью. К сожалению, этого не получается, причем наименее точно определяется величина $\gamma_{00}$. Именно поэтому в [2] значения $D, b$ и $a$ были рассчитаны из фиксированных значений $L_{00}$ и $\Theta_{00}$ и различных значений $\gamma_{00}$, которые известны из литературы. При этом возникала неопределенность в выборе значения $\gamma_{00}$ и вытекающих из этого расчета набора параметров $b$ и $a$.

Это привело к тому, что в работе [3] нами был предложен метод, в котором при данных значениях $r_{o}, D$ и $b$ величина $a$ корректировалась так, чтобы получить хорошее совпадение с экспериментальным значением коэффициента теплового расширения: $\alpha_{p}(P, T)=(\partial \ln V / \partial T)_{P}$, измеренным при $P=0$ и $T=300 \mathrm{~K}$. Таким методом для ОЦК-Fе величина $a$ была скорректирована от $a=3.58$ [2] до $a=2.95$ [3].

Но для тугоплавких металлов (Mo, W, Nb) такая корректировка величины $a$ для получения хорошего согласия с величиной $\alpha_{p}(P=0, T=300 \mathrm{~K})$ оказалась недостаточной. Это было связано с тем, что для таких металлов приближенно измеряется не только значение $\gamma_{00}$, но и величина $\Theta_{00}$. Например, для Мо и $\mathrm{W}$ в литературе приводятся следующие значения:

$$
\begin{gathered}
\Theta_{00}(\mathrm{Mo}) / \mathrm{K}=450[10], \quad 460-474.5[11], \\
472.4-474.9[12], \quad 259 \pm 11[13], \quad 423[14], \\
273.7[15], \quad 375-527[16], \quad 455-470[17] ; \\
\Theta_{00}(\mathrm{~W}) / \mathrm{K}=400[10], \quad 382.58-390[11], \\
382.6-384.6[12], \quad 384-388[13], \quad 383[14], \\
232[15], \quad 378 \pm 7[18] .
\end{gathered}
$$

Для $\mathrm{Nb}$ и Та разброс чуть меньше:

$$
\Theta_{00}(\mathrm{Nb}) / \mathrm{K}=275[10], \quad 275.7-277.05[11],
$$

$276.6-278.7[12], \quad 241 \pm 13[13], \quad 276[14], \quad 300[15]$. $\Theta_{00}(\mathrm{Ta}) / \mathrm{K}=240$ [10], 258-263.7 [11], 263.4-264.6 [12], 247 [13], 245 [14], $\quad 160.9$ [15].

По этим причинам в $[5,7]$ при расчетах свойств $\mathrm{W}$ и $\mathrm{Au}$ корректировались уже два параметра $b$ и $a$, 
при фиксированных значениях $r_{o}$ и $D$. Корректировка производилась до получения наилучшего совпадения как с экспериментальным значением коэффициента теплового расширения: $\alpha_{p}(P=0, T=300 \mathrm{~K})$, так и с экспериментальной зависимостью уравнения состояния $P\left(300 \mathrm{~K}, v / v_{o}\right)$. Здесь $v=V / N-$ удельный объем, $v_{o}=\pi r_{o}^{3} /\left(6 k_{p}\right)$. Однако, полученные таким путем параметры потенциала (1) привели к низким значениям модуля упругости $B(P=0, T=300 \mathrm{~K})$.

Поэтому в работах $[4,6]$ оптимизация потенциала (1) проводилась уже по трем параметрам: $D, b$ и $a$, при фиксированном значении $r_{o}$. В этом методе стремились получить наилучшее согласие с экспериментальными данными для $\alpha_{p}(P=0, T=300 \mathrm{~K}), B(P=0, T=300 \mathrm{~K})$ и с экспериментальной зависимостью $P\left(300 \mathrm{~K}, v / v_{o}\right)$. Таким путем были получены параметры потенциала (1) для Мo [4] и $\mathrm{Nb}$ [6]. Эти параметры позволили получить хорошие зависимости для функций: $P\left(T, v / v_{o}\right), \alpha_{p}(P, T)$, $B(P, T)$, изохорной $\left(C_{v}\right)$ и изобарной теплоемкости: $C_{p}=C_{v}\left(1+\gamma \alpha_{p} T\right)$, температуры плавления $\left(T_{m}\right)$, а также производных этих функций по давлению. Однако, ввиду высокой величины $D$, рассчитанные значения энергии сублимации $L_{00}$ и поверхностной энергии $(\sigma)$ оказались много больше экспериментальных данных.

В связи с этим в данной работе предложен самосогласованный метод определения параметров межатомного потенциала (1) по термоупругим свойствам кристалла, который будет более корректнее, чем методы из [1-7].

\section{2. Метод определения параметров}

В новом методе оптимизация потенциала (1) идет по двум параметрам: $b$ и $a$, при фиксированных значениях $r_{o}$ и молярной энергии сублимации $L_{00}$. При этом значения $\Theta_{00}, \gamma_{00}$ изменяются в широких диапазонах. Для металлов эти величины равны:

$$
100<\Theta_{00}<600 \text { и } 1.1<\gamma_{00}<4.1 \text {. }
$$

Из рассчитанных по формулам (7) наборам значений $D, b$ и $a$ отбираются только такие, которые при расчете уравнения состояния: $P\left(300 \mathrm{~K}, v / v_{o}=0.8\right)$, модуля упругости: $B(P=0, T=300 \mathrm{~K})$ и коэффициента теплового расширения: $\alpha_{p}(P=0, T=300 \mathrm{~K})$, дают величины, входящие в интервал допустимых значений. Данные функции вычисляются по формулам полученным в [3] на основании модели кристалла Эйнштейна и приближения „взаимодействия только ближайших соседей“

$$
\begin{aligned}
P & =-\left(\frac{\partial f_{H}}{\partial v}\right)_{T} \\
& =\left[\frac{k_{n}}{6} D \cdot U^{\prime}(R)+3 k_{B} \Theta_{E} \cdot \gamma \cdot E_{w}\left(\frac{\Theta_{E}}{T}\right)\right] \frac{1}{v},
\end{aligned}
$$

$$
\begin{gathered}
B=-v\left(\frac{\partial P}{\partial v}\right)_{T} \\
=P+\left[\frac{k_{n}}{18} D \cdot U^{\prime \prime}(R)+3 k_{B} \Theta_{E} \cdot \gamma \cdot(\gamma-q) \cdot E_{w}\left(\frac{\Theta_{E}}{T}\right)\right. \\
\left.-3 N \cdot k_{B} \cdot \gamma^{2} \cdot T \cdot F_{E}\left(\frac{\Theta_{E}}{T}\right)\right] \frac{1}{v} . \\
\alpha_{p}=\gamma \frac{C_{v}}{V \cdot B}=\frac{\gamma \cdot C_{v}}{N \cdot B\left[\pi r_{o}^{3} /\left(6 k_{p}\right)\right]}\left(\frac{v_{o}}{v}\right) .
\end{gathered}
$$

Здесь $f_{H}$ - удельная (на атом) свободная энергия Гельмгольца для кристалла Эйнштейна, $\Theta_{E}$ - это температура Эйнштейна, которая связана с температурой Дебая соотношением [19]: $\Theta=(4 / 3) \Theta_{E}, R=r_{o} / c-$ относительная линейная плотность кристалла, $U(R)-$ функция потенциальной энергии, которая, в соответствии с (1), равна

$$
\begin{gathered}
U(R)=\frac{a R^{b}-b R^{a}}{b-a}, \\
U^{\prime}(R)=R\left[\frac{\partial U(R)}{\partial R}\right]=\frac{a b\left(R^{b}-R^{a}\right)}{b-a}, \\
U^{\prime \prime}(R)=R\left[\frac{\partial U^{\prime}(R)}{\partial R}\right]=\frac{a b\left(b R^{b}-a R^{a}\right)}{b-a}, \\
F_{E}(y)=\frac{E_{w}(y)=0.5+\frac{1}{[\exp (y)-1]},}{[\exp (y)-1]^{2}}, \quad C_{v}=3 N \cdot k_{B} \cdot F_{E}\left(\frac{\Theta_{E}}{T}\right), \\
q=\left(\frac{\partial \ln \gamma}{\partial \ln v}\right)_{T}=\gamma \frac{X_{w}\left(1+2 X_{w}\right)}{1+X_{w}} .
\end{gathered}
$$

Проверка данного метода была проведена на $\mathrm{Fe}$ c объемно-центрированной кубической (ОЦК) структурой и на Аu с гранецентрированной кубической (ГЦК) структурой. Были определены как параметры межатомного потенциала (1), так и вытекающие из этих параметров значения энергии сублимации: $L_{00}$, уравнения состояния: $P\left(300 \mathrm{~K}, v / v_{o}=0.8\right)$, и значения решеточных свойств кристалла: $\alpha_{p}, B, \Theta, \gamma$. Здесь $\sigma$ - удельная (на единицу площади) поверхностная энергия грани (100), выражение для которой имеет вид [20]:

$$
\sigma(R, T)=-\frac{k_{n} D R^{2}}{12 \alpha^{2 / 3} r_{o}^{2}} L_{E}(R, T),
$$

Здесь введены обозначения:

$$
\begin{gathered}
L_{E}(R, T)=U(R)+3 H_{w}(R, T), \\
H_{w}(R, T)=\frac{6 \gamma}{(b+2)}\left[\frac{k_{B} \Theta_{E}}{D k_{n}}\right] E_{w}\left(\frac{\Theta_{E}}{T}\right) .
\end{gathered}
$$

Таким образом, в данном методе производится оптимизация параметров потенциала (1) как по энергетическим, так и по упругим параметрам кристалла. 
Энергия сублимации, параметры межатомного потенциала (1) и статья, в которой они были определены, и свойства, которые получаются при расчете с использованием указанных параметров

\begin{tabular}{|c|c|c|c|c|c|c|c|c|c|c|c|c|}
\hline Кристалл & $\begin{array}{c}L_{00} \\
\mathrm{~kJ} / \mathrm{mol}\end{array}$ & $\begin{array}{l}D / k_{B} \\
\mathrm{~K}\end{array}$ & $b$ & $a$ & Статья & $\begin{array}{c}P(0.8) \\
\mathrm{GPa}\end{array}$ & $\begin{array}{c}\alpha_{p} \\
10^{-6} \mathrm{~K}^{-1}\end{array}$ & $\begin{array}{c}B=-v(\partial P / \partial v)_{T} \\
\mathrm{GPa}\end{array}$ & $B^{\prime}(P)=(\partial B / \partial P)_{T}$ & $\begin{array}{l}\Theta \\
\mathrm{K}\end{array}$ & $\gamma$ & $\begin{array}{c}\sigma, \\
10^{-3} \mathrm{~J} / \mathrm{m}^{2}\end{array}$ \\
\hline $\mathrm{Fe}$ & \begin{tabular}{|c|}
$413.80[1]$ \\
$-/ /-$ \\
414.454 \\
$\mathbf{4 1 4 . 6 3 4} \pm \mathbf{1 . 2 5 5}$ \\
{$[\mathbf{2 1}]$}
\end{tabular} & $\begin{array}{l}12561.53 \\
12576.70 \\
12576.70\end{array}$ & $\begin{array}{l}8.37 \\
8.26 \\
8.26\end{array}$ & $\begin{array}{l}3.097 \\
3.58 \\
2.95\end{array}$ & $\begin{array}{l}{[2]} \\
{[3]}\end{array}$ & $\begin{array}{c}75.9 \\
87.7 \\
71.1 \\
\mathbf{5 0 - 6 0} \\
{[\mathbf{2 3}]}\end{array}$ & $\begin{array}{c}34.1 \\
28.6 \\
36.1 \\
\mathbf{3 3}-\mathbf{3 8} \\
{[\mathbf{2 5 , 2 6}]}\end{array}$ & $\begin{array}{c}161.3 \\
185.7 \\
151.9 \\
\mathbf{1 5 6 - 1 7 1} \\
{[\mathbf{2 6 , 3 2}]}\end{array}$ & $\begin{array}{c}5.85 \\
5.98 \\
5.77 \\
\mathbf{4}-\mathbf{6} \\
{[\mathbf{2 6 , 3 2}]}\end{array}$ & $\begin{array}{c}415.17 \\
470.01 \\
399.5 \\
\mathbf{4 2 0}-\mathbf{4 7 8} \\
{[\mathbf{3 3}]}\end{array}$ & $\begin{array}{c}1.720 \\
1.701 \\
1.702 \\
\mathbf{1 . 4 - 1 . 8} \\
{[\mathbf{3 3}]}\end{array}$ & $\begin{array}{c}2202.98 \\
2207.35 \\
2204.95 \\
\mathbf{1 9 1 0}-\mathbf{2 5 2 5}[\mathbf{3 4}] \\
\mathbf{2 4 2 0}-\mathbf{2 4 8 0}[\mathbf{3 5}]\end{array}$ \\
\hline $\mathrm{Au}$ & \begin{tabular}{|c}
$368.19[1]$ \\
$-/ /-$ \\
$-/ /-$ \\
367.903 \\
$\mathbf{3 6 8 . 6 1 0} \pm \mathbf{1 . 2 5 5}$ \\
{$[\mathbf{2 1}]$}
\end{tabular} & $\begin{array}{c}7419.16 \\
7411.50 \\
-/ /- \\
-/ /-\end{array}$ & $\begin{array}{l}16.05 \\
15.56 \\
16.34 \\
12.82\end{array}$ & \begin{tabular}{|l|}
2.80 \\
1.96 \\
1.89 \\
2.93
\end{tabular} & $\begin{array}{l}{[2]} \\
{[2]} \\
{[7]}\end{array}$ & $\begin{array}{c}109.9 \\
71.9 \\
75.2 \\
80.5 \\
\mathbf{6 5}-\mathbf{7 5} \\
{[\mathbf{1 5}]}\end{array}$ & $\begin{array}{c}43.0 \\
64.0 \\
66.6 \\
41.7 \\
\mathbf{4 2 - 4 2 . 8} \\
{[\mathbf{2 5 , 2 7}]}\end{array}$ & $\begin{array}{c}166.4 \\
109.0 \\
109.4 \\
141.1 \\
\mathbf{1 6 6 . 4 - 1 7 3 . 2} \\
{[\mathbf{1 0}, \mathbf{1 4}]}\end{array}$ & $\begin{array}{c}8.35 \\
7.91 \\
8.15 \\
7.30 \\
\mathbf{5 . 3 1}[\mathbf{1 5}]-\mathbf{9 . 5 7}[\mathbf{2 7}]\end{array}$ & $\begin{array}{c}198.04 \\
156.23 \\
155.38 \\
190.09 \\
\mathbf{1 5 6}-\mathbf{1 7 8} \\
{[\mathbf{3 3}]}\end{array}$ & $\begin{array}{c}3.001 \\
2.921 \\
3.051 \\
2.464 \\
\mathbf{2 . 3}-\mathbf{3 . 0 5} \\
{[\mathbf{3 3}]}\end{array}$ & $\begin{array}{c}1531.38 \\
1523.82 \\
1523.08 \\
1530.31 \\
\mathbf{1 1 7 5}-\mathbf{1 8 5 0}[\mathbf{3 4}] \\
\mathbf{1 5 0 0}-\mathbf{1 5 1 0}[\mathbf{3 5}]\end{array}$ \\
\hline $\mathrm{Nb}$ & \begin{tabular}{|c|}
$719.65[1]$ \\
$-/ /-$ \\
$-/ /-$ \\
1001.25 \\
$\mathbf{7 1 9 . 4 7 2} \pm \mathbf{4 . 1 8 4}$ \\
{$[\mathbf{2 1}]$}
\end{tabular} & $\begin{array}{c}21732.20 \\
21706.44 \\
-/ /- \\
30200.0\end{array}$ & $\begin{array}{l}9.24 \\
5.81 \\
7.50 \\
6.2\end{array}$ & \begin{tabular}{|l|}
2.55 \\
1.88 \\
1.72 \\
2.3
\end{tabular} & $\begin{array}{l}{[2]} \\
{[2]} \\
{[6]}\end{array}$ & $\begin{array}{c}77.7 \\
31.1 \\
39.0 \\
57.4 \\
\mathbf{5 0 - 6 0} \\
{[\mathbf{1 5}]}\end{array}$ & $\begin{array}{c}24.0 \\
37.0 \\
38.4 \\
20.6 \\
\mathbf{2 1 . 3 - 2 2 . 8} \\
{[\mathbf{2 5 , 2 8 , 2 9}]}\end{array}$ & $\begin{array}{c}167.2 \\
77.2 \\
90.5 \\
145.1 \\
\mathbf{1 4 4 . 2}-\mathbf{1 7 0 . 2} \\
{[\mathbf{1 0}, \mathbf{1 4}]}\end{array}$ & $\begin{array}{c}5.95 \\
4.58 \\
5.09 \\
4.84 \\
\mathbf{3 . 3}[\mathbf{1 5}]-\mathbf{1 4 . 5}[\mathbf{1 4}]\end{array}$ & $\begin{array}{c}327.28 \\
237.02 \\
236.38 \\
331.56 \\
\mathbf{2 4 1}[\mathbf{1 3}]-\mathbf{3 0 0}[\mathbf{1 0}]\end{array}$ & $\begin{array}{c}1.869 \\
1.300 \\
1.581 \\
1.367 \\
\mathbf{1 . 3}-\mathbf{1 . 6 9} \\
{[\mathbf{3 3}]}\end{array}$ & $\begin{array}{c}2870.66 \\
2860.54 \\
2859.80 \\
3998.34 \\
\mathbf{2 0 5 0}-\mathbf{2 5 5 0}[\mathbf{3 4}] \\
\mathbf{2 6 6 0}-\mathbf{2 7 0 0}[\mathbf{3 5}]\end{array}$ \\
\hline $\mathrm{Ta}$ & \begin{tabular}{|c}
$706.26[1]$ \\
$-/ /-$ \\
$-/ /-$ \\
$785.608[21]$ \\
$\mathbf{7 8 5 . 6 0 8} \pm \mathbf{4 . 1 8 4}$ \\
{$[\mathbf{2 1}]$}
\end{tabular} & $\begin{array}{c}21318.49 \\
21305.51 \\
-/ /- \\
23701.02\end{array}$ & $\begin{array}{r}7.86 \\
7.01 \\
11.16 \\
7.92\end{array}$ & \begin{tabular}{|l|}
3.49 \\
2.90 \\
2.52 \\
3.13
\end{tabular} & $\begin{array}{l}{[2]} \\
{[2]}\end{array}$ & $\begin{array}{c}86.3 \\
60.8 \\
97.9 \\
85.8 \\
\mathbf{5 5}-\mathbf{7 5} \\
{[\mathbf{1 5 , 2 4}]}\end{array}$ & $\begin{array}{c}18.5 \\
23.2 \\
24.8 \\
18.6 \\
\mathbf{1 8}-\mathbf{1 9 . 8} \\
{[\mathbf{2 5 , 2 9 , 3 0}]}\end{array}$ & $\begin{array}{c}192.8 \\
142.4 \\
194.7 \\
193.8 \\
\mathbf{1 9 2}[\mathbf{1 5}]-\mathbf{1 9 5}[\mathbf{2 4}]- \\
\mathbf{2 0 1 . 9}[\mathbf{1 4}]\end{array}$ & $\begin{array}{c}5.80 \\
5.32 \\
6.58 \\
5.70 \\
\mathbf{3 . 4 - 3 . 5 5}[\mathbf{2 4}] \\
\mathbf{- 3 . 8 4 2}[\mathbf{1 4}]\end{array}$ & $\begin{array}{c}289.93 \\
244.43 \\
242.84 \\
278.54 \\
\mathbf{1 6 0 . 9}[\mathbf{1 5}] \\
\mathbf{- 2 6 4 . 6}[\mathbf{1 2}]\end{array}$ & $\begin{array}{c}1.640 \\
1.499 \\
2.190 \\
1.650 \\
\mathbf{1 . 5}-\mathbf{2 . 1 9} \\
{[\mathbf{3 3}]}\end{array}$ & $\begin{array}{c}2819.56 \\
2815.53 \\
2814.5 \\
3136.47 \\
\mathbf{1 9 5 0}-\mathbf{2 6 8 0}[\mathbf{3 4}] \\
\mathbf{2 9 0 0}-\mathbf{3 1 5 0}[\mathbf{3 5}]\end{array}$ \\
\hline Mo & \begin{tabular}{|c|}
$655.31[22]$ \\
$-/ /-$ \\
1624.34 \\
$\mathbf{6 5 4 . 8 6 7} \pm \mathbf{2 . 9 2 8}$ \\
{$[\mathbf{2 1}]$} \\
\end{tabular} & \begin{tabular}{|c|}
19832.34 \\
19776.91 \\
48950.0
\end{tabular} & $\begin{array}{l}8.29 \\
7.68 \\
6.82\end{array}$ & \begin{tabular}{|l|}
3.93 \\
1.93 \\
1.87 \\
\end{tabular} & $\begin{array}{l}{[2]} \\
{[4]}\end{array}$ & $\begin{array}{r}115.0 \\
48.4 \\
97.5 \\
\mathbf{8 0}-\mathbf{9 5} \\
{[\mathbf{1 5 , 1 7}]} \\
\end{array}$ & $\begin{array}{c}16.4 \\
37.2 \\
14.9 \\
\mathbf{1 4 - 1 6 . 5} \\
{[\mathbf{2 5 , 2 9 , 3 1 ]}}\end{array}$ & $\begin{array}{c}248.7 \\
110.6 \\
242.6 \\
\mathbf{2 4 4 - 2 6 0} \\
{[\mathbf{1 7}]}\end{array}$ & $\begin{array}{c}6.09 \\
5.22 \\
4.90 \\
4.21-4.67 \\
{[17]}\end{array}$ & $\begin{array}{c}451.13 \\
253.91 \\
383.66 \\
\mathbf{4 5 5}-\mathbf{4 7 0} \\
{[\mathbf{1 7}]}\end{array}$ & $\begin{array}{c}1.710 \\
1.610 \\
1.468 \\
\mathbf{1 . 9 5 - 2 . 0 3} \\
{[\mathbf{1 7}]} \\
\end{array}$ & \begin{tabular}{|c|}
2907.50 \\
2889.24 \\
7207.60 \\
$\mathbf{1 8 6 5}-\mathbf{2 6 3 0}[\mathbf{3 4}]$ \\
$\mathbf{2 9 1 0}-\mathbf{3 0 0 0}[\mathbf{3 5}]$
\end{tabular} \\
\hline W & \begin{tabular}{|c|}
$848.10[1]$ \\
$-/ /-$ \\
847.987 \\
$\mathbf{8 5 5 . 6 4 4} \pm \mathbf{4 . 1 8 4}$ \\
{$[\mathbf{2 1}]$}
\end{tabular} & $\begin{array}{c}25594.79 \\
25608.93 \\
-/ /-\end{array}$ & $\begin{array}{l}8.82 \\
8.58 \\
6.28\end{array}$ & \begin{tabular}{|l|}
3.45 \\
4.06 \\
3.97
\end{tabular} & $\begin{array}{l}{[2]} \\
{[5]}\end{array}$ & $\begin{array}{c}136.2 \\
157.6 \\
103.1 \\
\mathbf{1 0 3}-\mathbf{1 2 0} \\
{[\mathbf{1 5}]}\end{array}$ & $\begin{array}{c}15.0 \\
12.6 \\
13.6 \\
\mathbf{1 1}-\mathbf{1 5} \\
{[\mathbf{1 8 , 2 5 , 2 9 ]}}\end{array}$ & $\begin{array}{c}295.7 \\
339.6 \\
243.5 \\
\mathbf{2 9 6}[\mathbf{1 5}] \mathbf{- 3 2 3 . 3}[\mathbf{1 0}]\end{array}$ & $\begin{array}{c}6.10612 \\
6.22903 \\
5.42890 \\
\mathbf{4 . 4}[\mathbf{1 5}]-\mathbf{1 9 . 1}[\mathbf{1 4}]\end{array}$ & $\begin{array}{c}330.35 \\
381.042 \\
393.482 \\
\mathbf{2 3 2}[\mathbf{1 5}]-\mathbf{4 0 0}[\mathbf{1 0}] \\
\mathbf{3 1 0}-\mathbf{6 1 0}[\mathbf{3 3}]\end{array}$ & $\begin{array}{c}1.800 \\
1.760 \\
1.377 \\
\mathbf{1 . 1}-\mathbf{1 . 7 6} \\
{[\mathbf{3 3}]}\end{array}$ & $\begin{array}{c}3715.79 \\
3719.21 \\
3718.11 \\
\mathbf{2 6 6 8}-\mathbf{2 7 1 2}[\mathbf{3 4}] \\
\mathbf{3 2 7 0}-\mathbf{3 6 8 0}[\mathbf{3 5}]\end{array}$ \\
\hline
\end{tabular}




\section{3. Результаты расчетов}

В таблице представлены полученные в различных работах параметры межатомного потенциала (1) и вытекающие из этих параметров значения указанных свойств как для ОЦК-Fe и ГЦК-Au, так и для тугоплавких металлов $\mathrm{Nb}$, Та, Мо и $\mathrm{W}$, которые имеют ОЦК структуру. Для координаты минимума потенциала (1) брали следующие значения: $r_{o} /\left[10^{-10} \mathrm{~m}\right]=2.4775$ (ОЦК-Fе), 2.8751 (ГЦК-Аu), 2.8648 (ОЦК-Nb), 2.8648 (ОЦК-Та), 2.7365 (ОЦК-W) - из [1]; 2.72 (ОЦК-Мо) - из [2]. Для каждого кристалла в верхних строках представлены результаты полученные новым методом, а в нижней строке представлены экспериментальные оценки указанных свойств. Для ОЦК-Та расчет параметров потенциала (1) и свойств сделан для двух значений энергий сублимации: из [1] и из [21], ввиду их заметного различия. Результаты представлены в первой и четвертой строках.

Как видно из таблицы, полученные новым методом параметры потенциала (1) для $\mathrm{Fe}$ и $\mathrm{Au}$ дают при $T=300 \mathrm{~K}$ и $P=0$ значения решеточных свойств лучше, чем потенциалы из других работ. Но величина $P(300 \mathrm{~K}$, $\left.v / v_{o}=0.8\right)$ по данным параметрам получилась несколько выше экспериментальных данных. Это указывает на то, что полученные здесь параметры потенциала работоспособны в области упругих деформаций, т.е. при $v / v_{o} \geq 0.9$. Аналогичный вывод можно сделать и по результатом расчета свойств тугоплавких ОЦКметаллов. Но потенциальные параметры, полученные предлагаемым методам, дают в комплексе более лучшее согласие с набором экспериментальных данных: как по $L_{00}$ и $P\left(300 \mathrm{~K}, v / v_{o}=0.8\right)$, так и для определенных при $T=300 \mathrm{~K}$ и $P=0$ решеточных свойств: $\alpha_{p}, B, \Theta, \gamma$ и $\sigma$. Для ОЦК-Та лучшее согласие рассчитанных свойств с экспериментальными данными получено из значения энергии сублимации из [21], которые представлены в четвертой строке.

Помимо определения параметров потенциала (1) данный метод расчета позволяет определить более достоверные значения для таких свойств как $\Theta, \gamma$ и $\sigma$, которые для тугоплавких металлов имеют большой разброс экспериментальных данных. Как было указано в $[9,34,36]$ современные экспериментальные методы не позволяют измерить данные параметры с необходимой точностью (см. разброс экспериментальных данных в таблице). Кроме этого, на примере ОЦК-Та показано, что данный метод может быть использован для выбора более достоверного значения энергии сублимации кристалла. Поэтому данный комплексный метод расчета параметров потенциала (1), также позволяет более точнее определять и термодинамические свойства кристалла.

\section{4. Заключение}

Предложен новый метод для определения 4-х параметров потенциала Ми-Леннард-Джонса по термоупругим свойствам кристалла. Показано, что данный метод более корректен, чем известные из литературы другие методы определения параметров потенциала. Определены параметры межатомного потенциала для ГЦК-Аu, и для кристаллов $\mathrm{Fe}, \mathrm{Nb}, \mathrm{Ta}, \mathrm{Mo}, \mathrm{W}$, имеющих ОЦК-структуру. На основании полученных результатов указаны более достоверные значения для $\Theta, \gamma$ и $\sigma$ указанных кристаллов. На примере ОЦК-Та показано, что данным методом можно также указать более достоверное значение энергии сублимации кристалла.

\section{Благодарности}

Автор выражает благодарность С.П. Крамынину, Н.Ш. Газановой и 3.М. Сурхаевой за плодотворные дискуссии и помощь в работе.

\section{Финансирование работы}

Работа выполнена при финансовой поддержке РФФИ (грант № 18-29-11013_мк) и Программы Президиума РАН (программа № 6, грант 2-13).

\section{Конфликт интересов}

Автор заявляет, что у него нет конфликта интересов.

\section{Список литературы}

[1] S. Zhen, G.J. Davies. Phys. Status Solidi A 78, 2, 595 (1983). DOI: $10.1002 /$ pssa.2210780226

[2] М.Н. Магомедов. Теплофизика высоких температур 44, 4, 518 (2006). DOI: $10.1007 / \mathrm{s} 10740-006-0064-$

[3] М.Н. Магомедов. ЖТФ 85, 11, 48 (2015). DOI: $10.1134 / \mathrm{S} 1063784215110195$

[4] E.N. Akhmedov. J. Phys. Chem. Solids 121, 62 (2018). DOI: 10.1016/j.jpcs.2018.05.011

[5] N.Sh. Gazanova. Appl. Solid State Chem. 3, 4, 36 (2018). DOI: 10.18572/2619-0141-2018-3-4-36-40

[6] S.P. Kraminin, E.N. Ahmedov. J. Phys. Chem. Solids 135, 109108 (2019). DOI: 10.1016/j.jpcs.2019.109108

[7] E.N. Ahmedov. J. Phys.: Conf. Ser. 1348, 012002, 1 (2019). DOI: $10.1088 / 1742-6596 / 1348 / 1 / 012002$

[8] Э.А. Мелвин-Хьюз. Физическая химия. ИЛ, М. (1962). 1148 c. [E.A. Moelwyn-Hughes. Physical Chemistry. Pergamon Press, London (1961).]

[9] М.Н. Магомедов. ФТТ 45, 1, 33 (2003). DOI: $10.1134 / 1.1537405$

[10] Ч. Киттель. Введение в физику твердого тела. Наука, М. (1978). 792 c. [Ch. Kittel. Introduction to Solid State Physics, J. Wiley and Sons Ltd., N.-Y. (1976).]

[11] M.M. Shukla, N.T. Padial. Rev. Brasil. Fís. 3, 1, 39 (1973). http://sbfisica.org.br/bjp/download/v03/v03a03.pdf

[12] J.K.D. Verma, M.D. Aggarwal. J. Appl. Phys. 46, 7, 2841 (1975). DOI: $10.1063 / 1.322028$

[13] В.Е. Зиновьев. Теплофизические свойства металлов при высоких температурах. Справочник. Металлургия, М. (1989). 384 c. 
[14] Физические величины. Справочник / Под. ред. И.С. Григорьева, Е.3. Мейлихова. Энергоатомиздат, М. (1991). 1232 c. [Handbook of Physical Quantities Ed. I.S. Grigoriev, E.Z. Meilikhov. Energoatomizdat, M. (1991); CRC Press, Boca Raton, Florida (1996).]

[15] A. Karbasi, S.K. Saxena, R. Hrubiak. CALPHAD: Comp. Coupling Phase Diagrams Thermochem. 35, 1, 72 (2011). DOI:10.1016/j.calphad.2010.11.007

[16] P.D. Desai. J. Phys. Chem. Ref. Data 16, 1, 91 (1987). DOI: $10.1063 / 1.555794$

[17] X. Huang, F. Li, Q. Zhou, Y. Meng, K.D. Litasov, X. Wang, B. Liu, T. Cui. Sci. rep. 6, 19923 (2016). DOI: $10.1038 /$ srep 19923

[18] В.Ю. Бодряков. Теплофизика высоких температур 53, 5, 676 (2015). DOI: 10.7868/S0040364415040067

[19] Л. Жирифалько. Статистическая физика твердого тела. Мир, М. (1975). 383 c. [L.A. Girifalco. Statistical Physics of Materials. J. Wiley and Sons Ltd, N.-Y. (1973).]

[20] М.Н. Магомедов. Кристаллография 62, 3, 487 (2017). DOI: $10.1134 /$ S1063774517030142

[21] http://www.chem.msu.su/cgi-bin/tkv.pl.

[22] Термические константы веществ. Справочник в 10-ти вып. / Под ред. В.П. Глушко, ВИНИТИ, М. (1965-1982).

[23] Д.К. Белащенко, О.И. Островский. ЖФХ 85, 6, 1063 (2011). DOI: $10.1134 / \mathrm{S} 0036024411060094$

[24] J.-B. Gu, C.-J. Wang, W.-X. Zhang, B. Sun, G.-Q. Liu, D.D. Liu, X.-D. Yang. Chin. Phys. B 25, 12, 126103 (2016). DOI: $10.1088 / 1674-1056 / 25 / 12 / 126103$

[25] С.И. Новикова. Тепловое расширение твердых тел. Наука, M. (1974). 294 c.

[26] D.R. Wilburn, W.A. Bassett. Am. Mineral. 63, 5-6, 591 (1978). https://pubs.geoscienceworld.org/msa/ammin/articleabstract/63/5-6/591/40926

[27] M.G. Pamato, I.G. Wood, D.P. Dobson, S.A. Hunt, L. Voŏadlo. J. Appl. Crystallography 51, 2, 470 (2018). DOI: $10.1107 / \mathrm{S} 1600576718002248$

[28] M.E. Straumanis, S. Zyszczynski. J. Appl. Crystallography 3, 1, 1 (1970). DOI: 10.1107/s002188987000554x

[29] K. Wang, R.R. Reeber. Mater. Sci. Engineering: Rep. 23, 3, 101 (1998). DOI: 10.1016/s0927-796x(98)00011-4

[30] В.Ю. Бодряков. Теплофизика высоких температур 54, 3, 336 (2016). DOI: 10.7868/S0040364416030029

[31] В.Ю. Бодряков. Теплофизика высоких температур 52, 6, 863 (2014). DOI: 10.7868/S004036441404005X

[32] Y. Shibazaki, K. Nishida, Y. Higo, M. Igarashi, M. Tahara, T. Sakamaki, H. Terasaki, Y. Shimoyama, S. Kuwabara, Y. Takubo, E. Ohtani. Am. Mineral. 101, 5, 1150 (2016). DOI: 10.2138/am-2016-5545

[33] М.Н. Магомедов. Изучение межатомного взаимодействия, образования вакансий и самодиффузии в кристаллах. Физматлит, М. (2010). 544 с.

[34] V.K. Kumikov, Kh.B. Khokonov. J. Appl. Phys. 54, 3, 1346 (1983). DOI: $10.1063 / 1.332209$

[35] Q. Jiang, H.M. Lu, M. Zhao. J. Phys.: Condens. Matter 16, 4, 521 (2004). DOI: 10.1088/0953-8984/16/4/001

[36] М.Н. Магомедов. ЖТФ 80, 9, 150 (2010). DOI: $10.1134 / \mathrm{S} 1063784210090240$

Редактор Т.Н. Василевскал 